\title{
A semantic event based framework for complex situations modeling and identification in smart environments
}

\author{
Abderrahim Lakehal $^{{ }^{*}}$, Adel Alti ${ }^{2}$ and Philippe Roose ${ }^{3}$ \\ Ph.D. Student, LRSD, University Ferhat Abbas Setif-1, Algeria ${ }^{1}$ \\ Associate Professor, LRSD, University Ferhat Abbas Setif-1, Algeria ${ }^{2}$ \\ Associate Professor, LIUPPA, University of PAU, Anglet, France ${ }^{3}$
}

Received: 11-September-2018; Revised: 1-December-2018; Accepted: 30-January-2019

(C)2019 Abderrahim Lakehal et al. This is an open access article distributed under the Creative Commons Attribution (CC BY) License, which permits unrestricted use, distribution, and reproduction in any medium, provided the original work is properly cited.

\begin{abstract}
Integration of technologies and smart services is an essential need in most pervasive smart automation systems, especially those requiring agile situation management such as a smart home. Ontology reasoning is an efficient technique for identifying the situation of such systems by capturing the context and optimizing connected object lifecycle. It is based on common-sense knowledge for interpreting a huge number of events from different and heterogeneous connected objects. The ontology-driven approach may be used to solve the parallel incoming events using new combination operators in order to achieve high accuracy rates. This paper presents a flexible, modular and hierarchical loosely coupled framework based on open semantic services in order to identify highly complex events to hold the situations with high efficiency. The proposed approach is evaluated on smart domains use cases and compared with other existing methods. Results show interesting ratios of situation identification accuracy with low execution time.
\end{abstract}

\section{Keywords}

Emergency situation, IoT, Multi-layered ontology, Smart context manager.

\section{Introduction}

With the advancement of technologies and smart services, a huge amount of context data (like images, audios, videos) is transmitted via the field of smart-* (Home, City, Office, Health, Tourism, etc.). This rapid development of smart technology makes easy access, distribute and share context data among users. Many pervasive systems and applications require management of user's situations in an intelligent way. As an example, we can cite fire or intrusion situations in smart home automation systems. Designing an efficient situations management system in terms of low execution time and high accuracy rate can be achieved by delivering and analyzing relevant context data. Internet of Thing (IoT) provides new opportunities to deliver collected context data, but has a negative influence on interactive applications in the growth of smart objects. Such strong and multiple connections between heterogeneous physical things will increase the complexity of the system.

\footnotetext{
*Author for correspondence
}

Therefore, dynamic reconfiguration (add, update or migrate services) of these systems will increase the maintenance costs. This problem is related to a high (re)connectivity of wide smart objects that continuously evolve faster.

Nowadays, some middleware allows the adaptations of component-based mobile applications referring to the evolving needs of the users and the execution context by exploiting rules languages users [1-8]. But these works do not address the complexity for reacting rapidly to situations under several moving locations and deduction using combination operators (i.e. parallel, sequence periodic and aperiodic, negation, fusion, recurrence). In this paper, we take advantage of semantic technologies, IoT domain with a component-based approach in the field of smart- * (home, health, cities, vehicle, etc.) making it possible to provide a new design approach and intelligent situation management in highly reconfigurable pervasive environments. We define explicit equivalence relationships between heterogeneous smart objects and services that describe semantic features related to the context data for interpretation 
of multiple events (for example GPS sensor that provides coordinates is semantically linked directly to interpret presence detection event or user location change event). We exploit a variety of combination operators (parallel, sequence periodic and aperiodic, negation, fusion, and recurrence) among situations for describing complex situations. We present a novel generic multi-layered ontology-based complex situation model (Multi-OSCM) that describes complex user-centric situations and its explicit relations between them in hierarchical abstraction levels for easy management facilities. We exploit the power of the distributed event detection framework for identifying complex situations.

The rest of the paper is organized as follows: We illustrate the requirements for processing complex situations in smart environments with a motivating scenario in Section 2. Related works are given in Section 3. Semantic event-based framework for situation modeling and identification is described in Section 4. Details of experiments and results are given in Section 5. The conclusion is given in Section 6.

\section{Motivating scenario: a smart home}

Let us show a real-daily life scenario: Mr. Adam lives in a four-room smart home. Each room consists of several (simple or multimedia) smart objects, including a camera, motion sensor, noisy sensor and temperature sensor, and alarm actuator. Mr. Adam has wearable devices, such as a smartphone and a smart watch used to capture GPS coordinates and other context parameters. The system needs to manage parallel incoming events (e.g. high temperature of several rooms, user presence in each room ...etc.). Figure 1 illustrates the smart home with both wearable smart objects and multimedia smart objects. All these events sensed by these smart objects will be used to identify alarm situations (e.g. fire or intrusion situations). When Mr. Adam leaves his smart home, all the cameras can be used to detect that nobody is at home. If one of these cameras is busy by serving another service, the system can substitute it by GPS sensor or motion sensor to ensure the continuity of event capturing instead of depending on the availability of the camera sensor. At that moment the system triggers an intrusion alarm application and fire alarm application for his home security (each anomaly Mr. 'Adam' can be notified by an SMS).

This scenario raises several problems: we need an efficient management of complex events with a semantic description of heterogeneous context data (e.g. which data represents an event? How do we interpret it? For example, a presence detector (camera) can provide Boolean values as input (True/False), others may give you a vector of displacement (GPS), it is the same event, but the interpretation is very different. How do we exploit it to infer and to manage concurrent users' situations? Each user has situations to be identified in order to suit the fast evolution of context data. The user has a wide range of devices willing to help him to achieve the new unplanned events. This poses a significant challenge to the collection of relevant contextual information for immediate user interests and needs. How do we exploit it to infer and to manage concurrent users' activities? For that, how do we exploit different incoming events to infer and to manage concurrent users' situations?

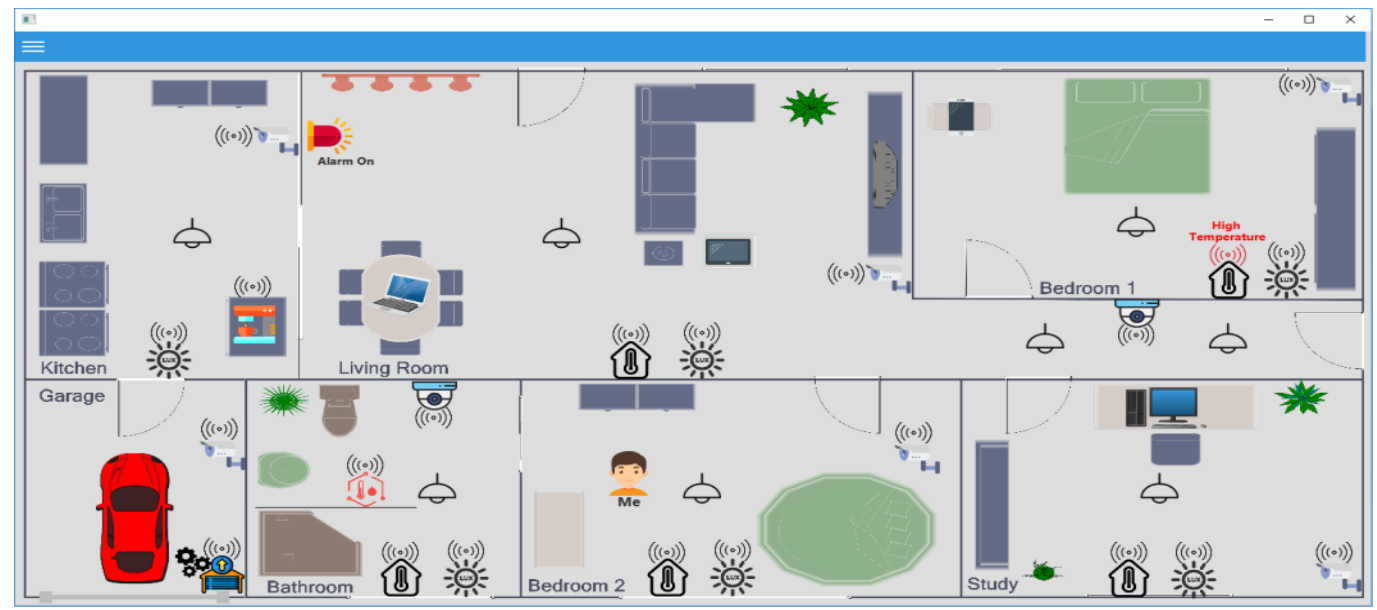

Figure 1 Smart home with different sensors installed 


\section{Related work}

Several components-based adaptation platforms were proposed to evolve continuously to keep up with users' needs and execution context changes such as: CybreMinder [1], Wcomp [2], Kalimucho-A [3], SensSocial [4], DiaSuite [7], CEMID [7], Dynamic Wright [9], event-condition-action rules [10], SHERLOCK [11]. WComp is a context-aware middleware for mobile devices, which was proposed by [2]. It is designed to provide mobile applications to users, to manage various context data collected from sensor devices and smartphones, connected objects according to the current users' needs, usage situations and execution context. This work reacts rapidly and efficiently to context changes, but has a lack of semantic mechanisms to process complex events at run-time and automatically generate component adaptation in top-down multimedia sensors from giving user's domain exploiting semantic constraints. Authors in [3] have proposed a context management middleware Kalimucho-A to provide services dedicated to the management of distributed context at a semantic level of the shared domain. This work offers an excellent smart service management and predefined policy deployment strategy, but disagrees in distributed situation inferring and complex event processing (e.g. Define "nobody at home" as abstract event aggregating all output values of motion sensors deployed in each room). SensSocial [4] was presented in order to integrate online social networks and mobile sensing data streams. This middleware is based on mobile social network sensors for capturing and filtering context data. It provides a common middleware to mobile social networks through which context streams from multiple remote devices can be aggregated and exploited. The user social contexts covered, allowing users to be aware of social events and the deliverance of richer contents from online social networks. This work does not take into account the semantics complex relations among events (e.g. two users interested to assist social events. The system detects events from crowdsourcing platforms and identifies relevant events according to their participation. The system notifies both users that each one of them will assist this event if and only if the participants are social media friends). Our work also introduces support to preserve service continuity features in users' domain space and distributed multimodal actions. The authors in [6] proposed an efficient mechanism to detect situations using ontology-based approach. However, these works do not hold the service, selection and then, they do not allow providing the appropriate service to the user.
Authors in [10] used ECA-based rules for allowing the application developers to easily create algorithms employing user context detection. Their work covers multi-dimensional context information, reasoning with various data collected from sensor devices and smartphones and supporting composite contexts. This work ensures better management of context-aware functionalities. The main drawback found is that it needs to be extended with complex context information processing with multiple mobile devices and lacks some high-level constructs that allow a developer to design more rich situations. Authors in [11] proposed SHERLOCK that handles continuously location-based services and the interactions between neighbor devices to provide up-to-date answers in heterogeneous and dynamic contexts. It provides a high semantic level approach to share knowledge among devices and enables the system to guide the user selecting the service that best fits the user needs in the given context. This work is efficient and shared between different services using an OWL. However, this work does not provide a support for managing complex situations at one time using a variety of combination operators and neither distributed multimodal actions. Authors in [12] proposed an ontology-based tool that assists users in detecting daily life activities and predicts some urgent situations. The proposed tool aims at collecting context data, inferring and reasoning over these data for the situation identification and decision making. The tool is based on inference rules provided by domain experts to generate appropriate services.

All of the above described related work use different techniques to deal with the heterogeneity and complexity of smart environments for achieving intelligent reconfigurable mobile applications. None of the works supports rich combination operators among events (i.e. parallel, sequence periodic and aperiodic, negation, fusion, recurrence). We adopted for a centralized/distributed and complex situation detection strategy at the semantic level (e.g. unambiguous interpretation of events using equivalence relationship among heterogeneous smart objects whatever its data format) in order to manage large context information according to the current user's needs that will give the application the flexibility and dynamicity. Our work is based on the component-oriented situation-related design for context-aware mobile distributed applications. Our improvements in this paper focus on two main points: (1) - the combination of events using operators such as parallel, sequence for identifying and deducing situations, (2) -the dynamic redeployment that will 
give the application the flexibility and the dynamicity needed to run through shared user's domain and the Kali-Smart middleware [6], which allows dynamic reconfiguration of applications on desktops, laptops and mobile devices.

\section{The proposed framework}

\subsection{General architecture}

We built our framework on the Kali-smart platform (Autonomic Semantic-Based Context-Aware Adaptation Platform) [6]. Kali-Smart is a platform based on a distributed semantic context monitor in order to manage important context information according to the current user's needs when moving in his smart environment. However, Kali-smart is only a "Toolbox" and lacks support for efficiently managing the explosion of users' situations and relations among them, semantic crowd-sensing data for mobile applications and providing interactive services for user-centric situations.

The key features of the framework are: 1) - provide smart mechanisms that retrieve and aggregate contextual information gathered from different constrained smart objects and multiple pervasive sensing devices, 2) -enrich some specific high-level context (situations) into a top-level generic complex situation-based ontology structure, 3) - detect complex situations and provide all distributed multimedia services that help users to access documents. Figure 2 details our framework components. The architecture consists of the Autonomic Semantic Complex Situation Identifier and Service Controller that is designed to follow the key stages of the situation identification process for provisioning services regarding the context changes during execution. It consists of a context monitor, situation inference engine, a service controller and service reconfiguration.

1. Context User Monitor is responsible for verifying the user's context change that calls a Context User Manager.

2. Complex Situation Manager is responsible for making inferences based on the ontology information, determining the appropriate services according to the deduced situations.

3. Service Orchestration and Reconfiguration: is responsible for deploying appropriate services to the user. It ensures service continuity and performs reconfiguration changes at the available devices to orchestrate the best way to deploy the services.

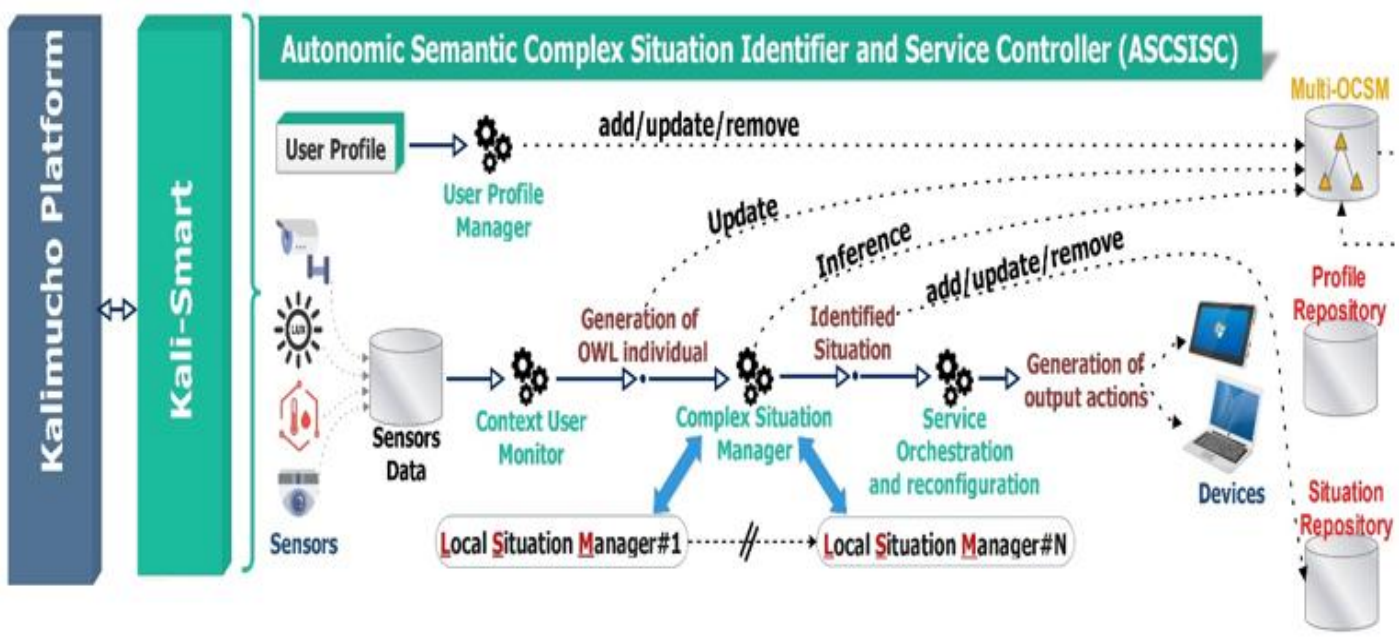

Figure 2 A semantic event-based framework for identification of a complex situation

\subsection{Multi-OCSM-Onto: multi-layers ontology for complex situation model}

Our ontology aims to describe heterogeneous mart objects as well as their various protocols (ZigBee, $3 \mathrm{G}, \mathrm{Wi}-\mathrm{Fi}$, etc.) and data formats (scalar, interval, Boolean, multimedia, features vector), and facilitates the hierarchical management of user complex situation mobile applications to better support context-awareness. Semantic reasoning based on a contextual semantic level between metadata constraints, and matching user profiles at the semantic level, with contextual information about heterogeneous context sources taken into account. Figure 3 presents an overview of our Multi-layered Ontology for Complex Situation Model for mobile applications (Multi-OCSM). This ontology is structured in six main classes, which are Smart Object class, Context class, Situation class, Service 
class Context Property class and Domain class. These classes represent generic concepts that can be used in any smart context-aware environments that aim at providing appropriate services to users according to their current situations.

- User-Domain Modeling. A domain (e.g. home domain, work domain, etc.) is composed of several devices. Each device is responsible for the execution of several services and each service may be contain one or more composite component. Each composite component has one or more atomic component which is deployed on specific smart devices. Each component provides a specific event and has hardware and software requirements in order to run in an optimal way.

- Context smart objects modeling. Our ontology can be used for modeling several heterogeneous sensors and actuators. It contains several sensors depending on its type and its features. These sensors serve as acquirers of raw contextual data which will be processed by reasoning techniques using common functionalities. We have two main types of sensors: logic and physical sensors. Physical sensors can include: bio-sensor (data captured by bio-sensors, like blood pressure, blood sugar and body temperature), weather sensor, environment sensor (data captured by environmental sensors, like room temperature, humidity, etc.), etc. logic sensors include device sensors (data captured by sensors, like CPU speed, battery energy), network sensor, calendar sensor, Gmail sensor, social media sensor, etc.

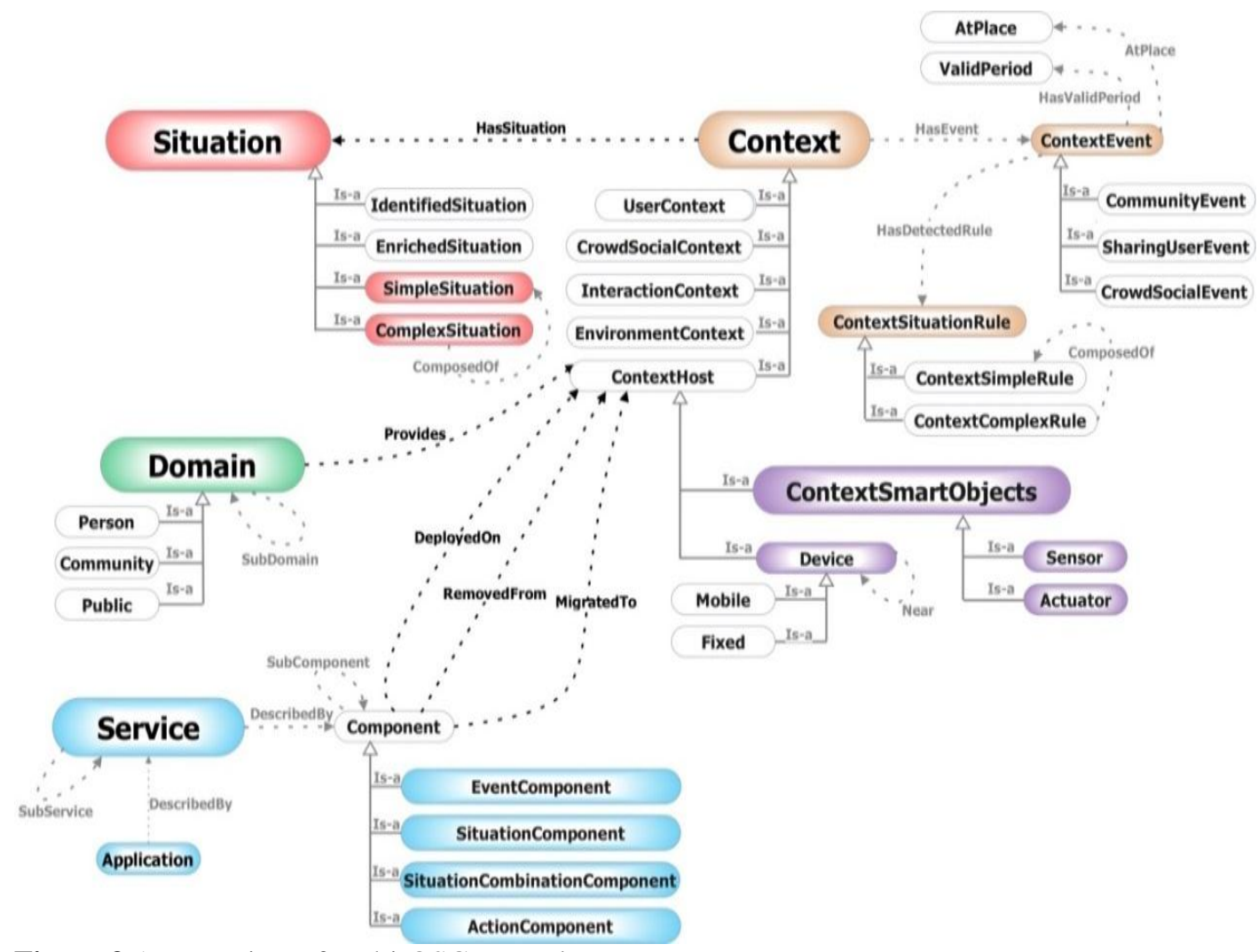

Figure 3 An overview of multi-OSCM ontology

- Context Modeling. The class Context enables the provision of adequate services to users based on their surrounding environments (Figure 3). It contains different context properties for each context category. For instance, the context related to the device has a set of properties (memory size, bandwidth, battery level, etc.). Each attribute can be used to represent the occurrence of an event.
We extend the work of [13] by two types of interpreting events: region, a vector of features (image, video, and audio). It helps wellinterpreting events, regardless of their sources using the qualitative parameters. Moreover, even in a specific context, for several smart domains (smart home, smart office), a parameter may have different interpretations. For instance, the 216 
parameter value "Presence" of the user corresponds to a vector of GPS coordinates if we consider smartwatch or corresponds to different features values if we consider Surveillance Camera and or corresponds to the Boolean value if we consider the motion sensor.

- Situation Modeling. The class situation represents the possible situations that we can define for each entity (user, device, and thing). Each situation is specified as a combination of sub-situations. A sub-situation corresponds to a contextual event. Within events, diverse relations, such as parallel, sequence, periodic and aperiodic, negation, fusion, and recurrence can be applied. Several actions can be triggered on behalf of the user as a result of situation identification. A complex situation is very useful for many concurrent users' activities where management is useful.

- Service Modeling. The distributed mobile application consists of a set of services. Each service is described by its distributed components on the concurrent. It is possible to adopt an application dynamically by reconfiguring its architecture. The common idea of our approach consists of reacting to user's situation evolutions and changing execution environment by dynamically adding/deleting/updating/ migrating components and connections between them.

4.3Mapping situations into dynamic semantic situation-aware service-oriented architecture

At the architectural level, we plan to model contextaware situation-based distributed mobile applications using a hierarchy semantic service-oriented architecture (e.g. event-based services, complex event-based- services, situation-based services, complex situation-based services, application services). Figure 4 depicts a representation of this architecture. An abstract view of distributed mobile application depicts it as a four-layered architecture of composite services. We distinguish two relationships: the first one between situations and event components are that the event components trigger the situations and are responsible for providing their high-level context data. The second is the deployable actions services that correspond to situations using a relation between a situation and services.

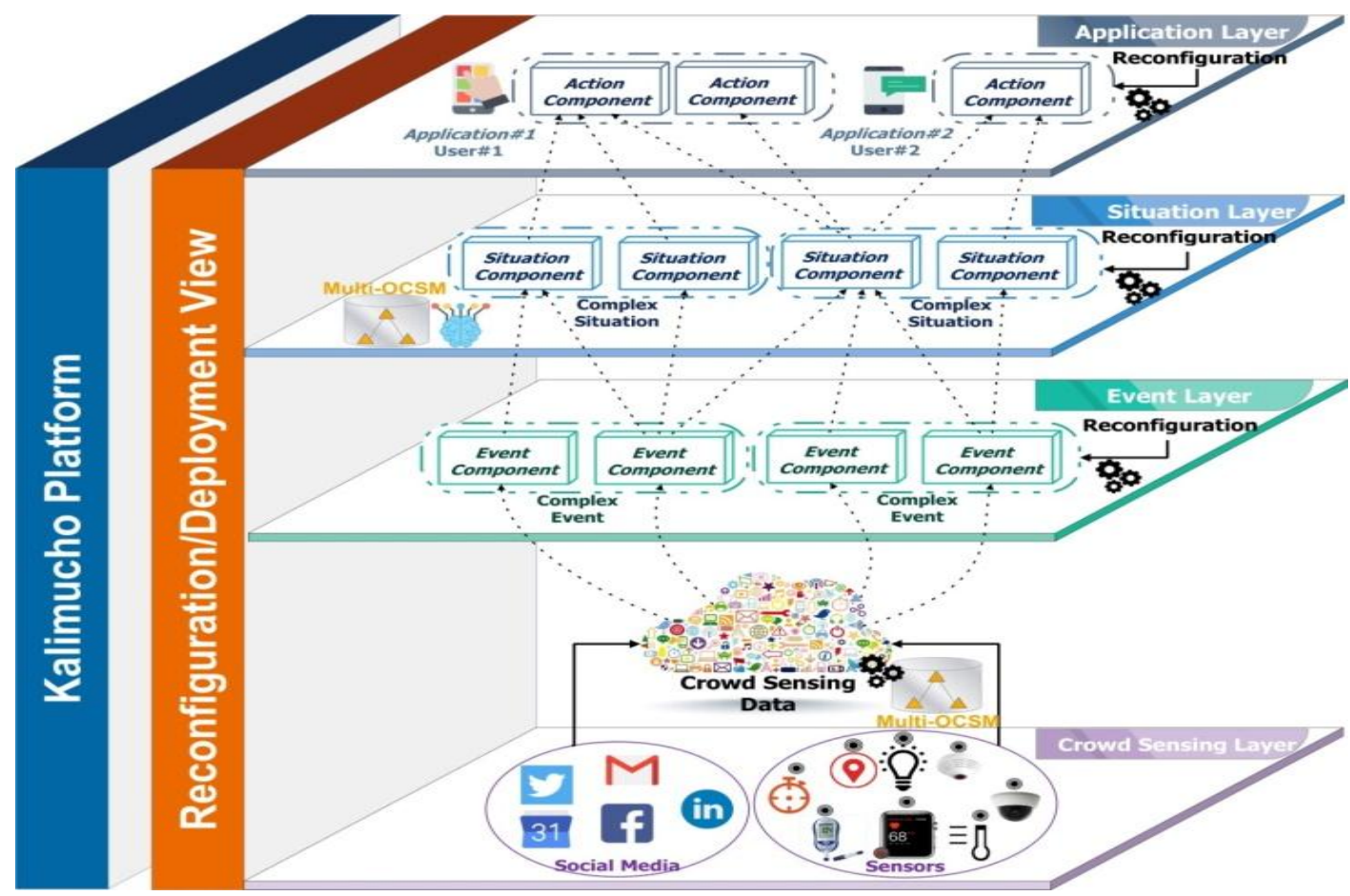

Figure 4 Mapping situation to semantic service-oriented model at architecture level

4.4Complex situation identification process

The identification of situation process flows can be is ensured by distributed semantic service context- aware architecture in 3-main step process (see Figure 5): 
- Firstly, distributed context monitors collect the context information from a user profile, several pervasive sensors (1) Each context monitor translates the input raw context data into OWL individuals of Multi-OCSM ontology 2. It's called the Complex Situation Manager of any significant event changes such as users' location changes, weather, and health changes.

- Secondly, according to identified events, changes, the Complex Situation Manager component refers to the "Situation Provider Registry" to launch the appropriate Simple Situation Manager (health, environment, city, home, etc.) for interrogating its local ontology. We use a set of spatial and temporal reasoning operators in order to deduce a list of possible situations. The temporal operators are SEQ, to describe sequential events, and OVERLAPS, to denote that two event occurrences happen during the same period. The spatial operators are distance operators (Equal, Near, Medium and Far) 3. Once the relevant situations are identified by several Simple Situation Manager, a list of identifying situations are stored in the ontology.

- Finally, Complex Situation Manager infers new complex situations from identified situations 4 and calls the Service controller and Reconfiguration component that generate the output reconfiguration scripts from the semantic link between the situation and services 5 . A set of services of the identified situation is deployed 6 and current services that match previous situations are removed silently.

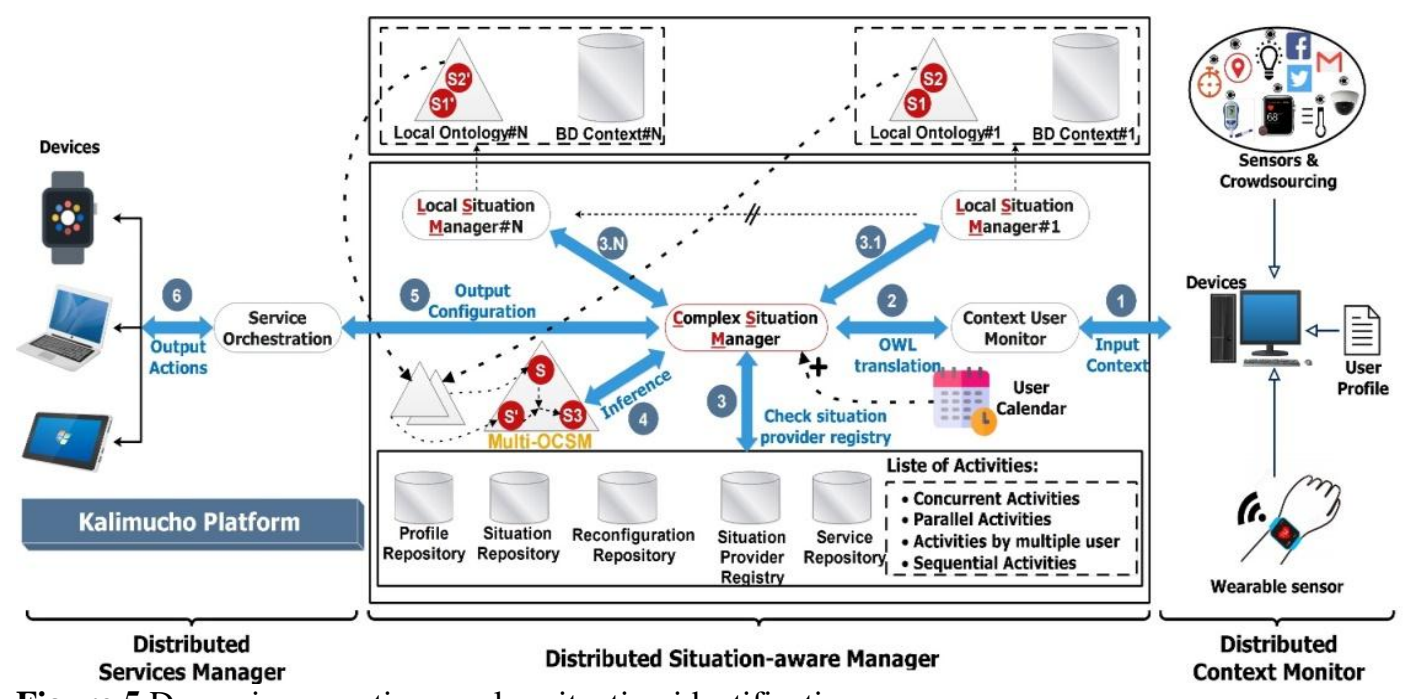

Figure 5 Dynamic semantic complex situation identification process

Figure 6 illustrates the mapping of home security complex situation into semantic services. The home security situation's description understood by the mobile application with the respect of user-defined constraints. It includes two parallel sub-situations: the home fire situation and the home intrusion situation. The home fire situation is identified by combining multiple events outputs (user location atomic event component, time atomic event component and homehigh-temperature complex event component). The GPS sensor of the smartwatch is used for detecting user location that is far from home. The complex event "home-high-temperature" is defined through three temperature atomic events outputs deployed on (kitchen, living-room and bedroom). Temperature sensors are enough to detect anomalies of high- temperature level in each room. Similarly, home intrusion situation is identified by combining multiple events components outputs (user location atomic event component, time atomic event component and "home-motion" complex event component). The complex event "home-motion" is defined through three motions sensors outputs deployed on (kitchen, living-room and bedroom). Motion sensors are enough to detect anomalies of intrusion. In case of identified of critical situations (like the home fire situation or home intrusion situation), the platform triggers an immediate response and request an urgent policy intervention where the coordinates of the home are determined and sent to the police station. To identify the home security situation as the parallel situation of two sub- 
situations (fire and intrusion situation), our framework must collect and analyze relevant context data to each sub-station (user location using GPS service, temperature level using temperature service, motion detection using motion service). Table 1 illustrates the event-based operators are used to infer situations that are related to the house security alarm situation.

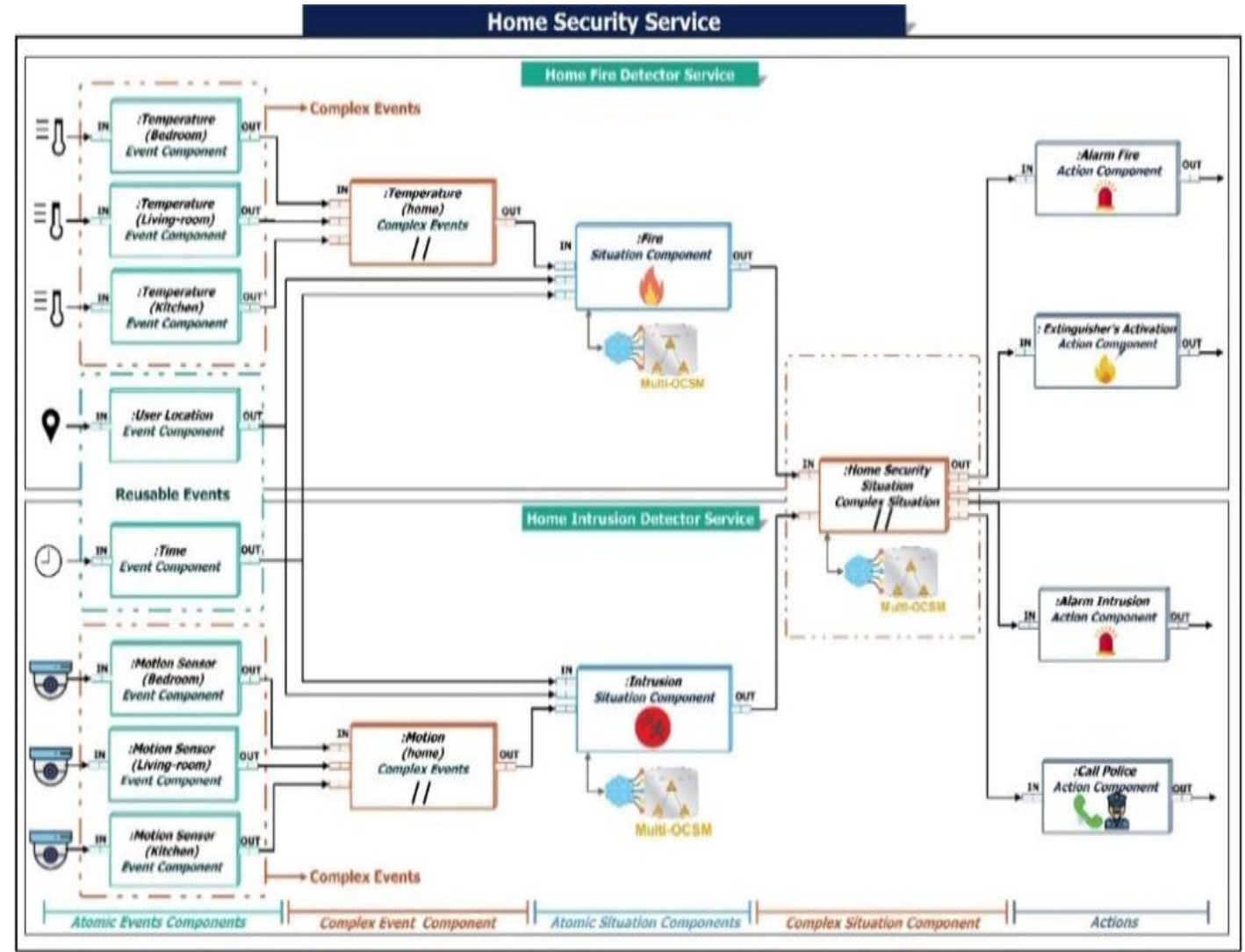

Figure 6 Home security complex situation and its associated services

Table 1 Description of some event-based operators

\section{Situation rules descriptions}

temperature is detected, send a message including home urgent situation

The home intrusion situation: Every time a sound volume is greater than $60 \%$ and an intrusion is detected, send a message including the home intrusion situation

The home security alarm situation: The home fire situation parallel with The home Intrusion situation

\section{Validation}

The framework is implemented in intellij IDEA 17.1 is able for managing complex situations and enriching user-related high-level context from sensed data for mobile application to cover all the situations responding to the user requirements. The evaluations are performed on are realized through a PC $3.4 \mathrm{GHz}$, 4Go RAM. The constraints of users increase from 10 to 60 in cases for OVERLAPS and SEQ operators. 219
Event-based operators' description

home_fire_situation $\equiv$ OVERLAPS (OVERLAPS

(Temperature_Bedroom_High, Temperature_ Livingroom_High), Temperature_Kitchen_High)

home_fire_situation $\equiv$ Far (Home) and

OVERLAPS (OVERLAPS (IsMotion_Bedroom, IsMotion_Livingroom), IsMotion_Kitchen)

home_security_situation $\equiv$

OVERLAPS (home_fire_situation, home_Intrusion_situation)

Figure 7 shows the execution time needed for identifying home security complex situations on increasing the number of contextual constraints. The results show that a huge amount of time is consumed. We improved the execution time by applying our proposed method that employs a smart situation manager to identify situations as explained in the proposed framework model (e.g. independent 
Abderrahim Lakehal et al.

constraints can be managed with parallel situations manager).

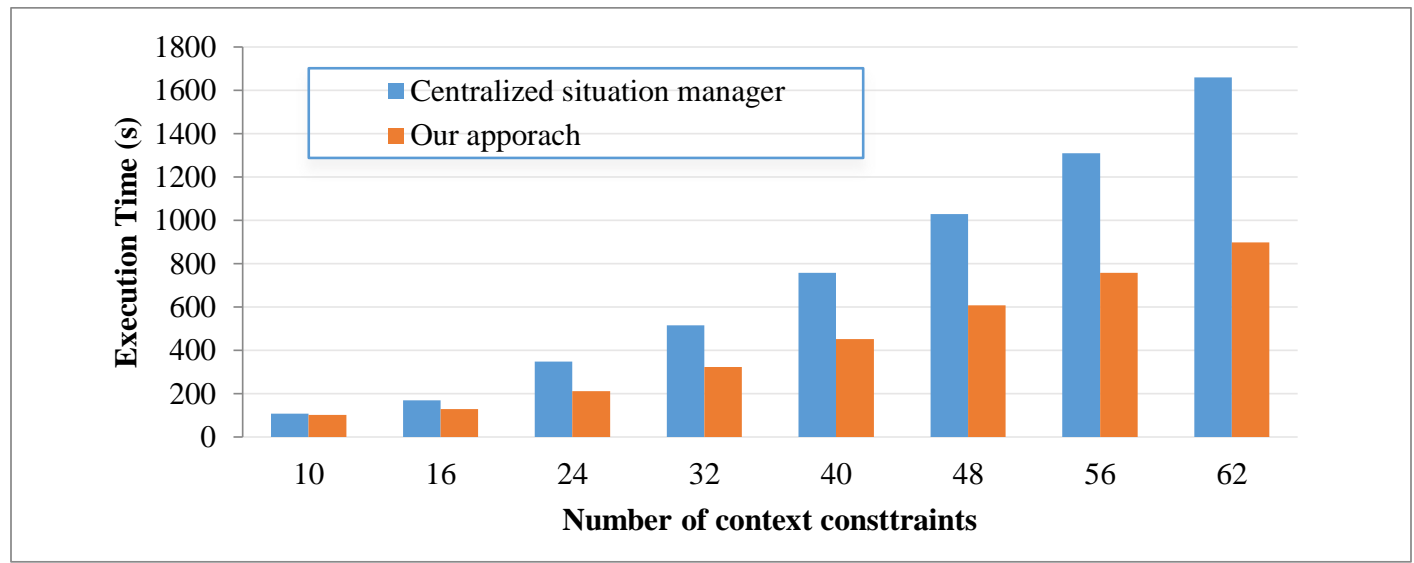

Figure 7 The execution time in different situations

\section{Conclusion}

This paper has proposed a new dynamic intelligent management framework based on multi-layered ontology model to the sensitive situations that identified in the field of smart- * (home, health, cities, vehicle, etc.). A novelty in this paper was a Complex Situation Manager component that infers and manages complex situations combined from multiple deduced atomic situation of various distributed situation manager based on specific smart domain in order to enhance performance when ensuring incoming parallel events. The proposed approach was investigated on Semantic Web technologies, IoT domain, with a component-based approach making it possible to provide new intelligent context management in highly reconfigurable pervasive environments. Experimental results had shown that the proposed method was good for a set of urgent situations while maintaining high accuracy rates and low execution time. We plan to improve the current work by applying a global framework using really simple and multimedia sensor devices (Arduino, Raspberry PI computer), smart objects and web technologies.

\section{Acknowledgment}

None.

\section{Conflicts of interest}

The authors have no conflicts of interest to declare.

\section{References}

[1] Dey AK, Abowd GD. Cybreminder: a context-aware system for supporting reminders. In international symposium on handheld and ubiquitous computing 2000 (pp. 172-86). Springer, Berlin, Heidelberg.

[2] Ferry N, Hourdin V, Lavirotte S, Rey G, Riveill M, Tigli JY. Wcomp, middleware for ubiquitous computing and system focused adaptation.2012.

[3] Da K, Dalmau M, Roose P. Kalimucho: middleware for mobile applications. In proceedings of the annual ACM symposium on applied computing 2014 (pp. 413-9). ACM.

[4] Mehrotra A, Pejovic V, Musolesi M. SenSocial: a middleware for integrating online social networks and mobile sensing data streams. In proceedings of the international middleware conference 2014 (pp. 20516). ACM.

[5] Alti A, Lakehal A, Laborie S, Roose P. Autonomic semantic-based context-aware platform for mobile applications in pervasive environments. Future Internet. 2016; 8(4):1-26.

[6] Angsuchotmetee C, Chbeir R, Cardinale Y, Yokoyama S. A pipelining-based framework for processing events in multimedia sensor networks. In proceedings of the annual symposium on applied computing 2018 (pp. 247-50). ACM.

[7] Bertran B, Bruneau J, Cassou D, Loriant N, Balland E, Consel C. DiaSuite: a tool suite to develop Sense/Compute/Control applications. Science of Computer Programming. 2014; 79:39-51.

[8] Chabridon S, Bouzeghoub A, Ahmed-Nacer A, Marie $\mathrm{P}$, Desprats T. Unified modeling of quality of context and quality of situation for context-aware applications in the internet of things. In International and Interdisciplinary Conference on Modeling and Using Context 2017 (pp. 370-4). Springer, Cham. 
[9] Allen R, Douence R, Garlan D. Specifying and analyzing dynamic software architectures. In international conference on fundamental approaches to software engineering 1998 (pp. 21-37). Springer, Berlin, Heidelberg.

[10] Papamarkos G. Event-condition-action rule languages over semistructured data (Doctoral dissertation, University of London).

[11] Yus R, Mena E, Ilarri S, Illarramendi A. SHERLOCK: semantic management of location-based services in wireless environments. Pervasive and Mobile Computing. 2014; 15:87-99.

[12] Gyrard A, Bonnet C, Boudaoud K, Serrano M. LOV4IoT: a second life for ontology-based domain knowledge to build semantic web of things applications. In 4th international conference on future internet of things and cloud (FiCloud) 2016 (pp. 25461). IEEE.

[13] Dromzée C, Laborie S, Roose P. A semantic generic profile for multimedia document adaptation. In intelligent multimedia technologies for networking applications: techniques and tools 2013 (pp. 225-46). IGI Global.

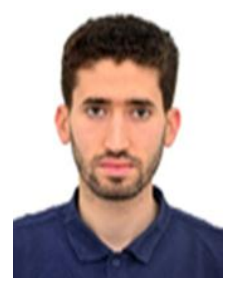

Lakehal Abderrahim is a Ph.D. student at the Computer Science Department, University of Ferhat Abbas Sétif 1, Setif, Algeria. $\mathrm{He}$ received his Master's degree in Computer Science in 2016 at the University of Ferhat Abbas Sétif 1, Setif, Algeria. He is a member of the Smart Context-aware Services Research Team of Network and Distributed System Laboratory. The subject of his thesis is Semantic Event-Based Modeling and Managing Complex Situations in smart environments.

Email: abderrahim.lakehal@univ-setif.dz

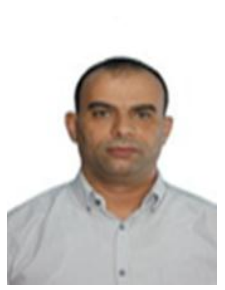

Adel Alti is an Associate Professor at the Computer Science Department, University of Ferhat Abbas Sétif 1, Setif, Algeria, since 2013. He received a Ph.D. degree in 2011 at the Computer Science Department from University Ferhat Abbas Setif-1, Algeria and his Postdoctoral in 2013 at the School of Computing, University of Biskra, Algeria. He is currently a Head of the Smart Context-aware Services Research Team of Network and Distributed System Laboratory. He supervised a number of $\mathrm{PhD}$ and Master Students. He published a number of book chapters and articles for international journals and conferences. His primary research interests include Mobility, Cloud Computing, Pervasif and Ubiquitous Computing, Context-Aware and Autonomic Applications.

Email: alti.adel@univ-setif.dz

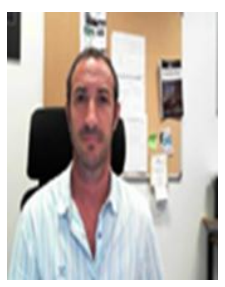

Phillipe Roose is an Associate Professor at LIUPPA researchLaboratory, Universityy of Pau, France. He is currently Director of the Computer Science Department and head of theT2I Research Team involving 17 permanents. He is coauthor of the Kalimucho platform (patented). He supervised $9 \mathrm{PhD}$ thesis and was involved in more than $30 \mathrm{PhD}$ defenses. He published many national and international articles, journals and books. His works are mainly focused on Middleware, Software Architecture, and Dynamic Adaptation, Context-Aware and Autonomic Applications

Email: philippe.roose@iutbayonne.univ-pau.fr 